\title{
Damage zones near excavations: plastic solution by means of stress trajectories
}

\author{
P. Haderka \& A. N. Galybin \\ Wessex Institute of Technology, Southampton, UK
}

\begin{abstract}
This study presents an alternative approach for identification of damaged zones near excavations. The approach is based on ideal plastic solutions but in contrast to the classical case it deals with the Cauchy's problem only by alternating classical solutions for slip zones with solutions for stress trajectories followed by conversion of the latter into slip grids. Comparisons with classical solutions are discussed.
\end{abstract}

Keywords: ideal plasticity, slip lines, stress trajectories.

\section{Introduction}

Damaged zones near excavations are frequently observed in underground mines. They present a significant issue for safety and effectiveness of mining operations and should be properly accounted for in design of particular excavations. This paper is aimed at the development of numerical methods for determination of damage zones in the case of long-wall excavations such as tunnels, well-bores, tabular stopes, etc. Another important motivation of this study is the application of a variant of the stress trajectory element method, STEM, which is currently under development in the Wessex Institute of Technology [1]. The method addresses the problem of stress identification in statically determined bodies by employing stress trajectories.

The concept of stress trajectories comes from photoelasticity, therefore one can adopt the following definition due to Frocht [2]: Stress trajectories are curves the tangents to which represent the directions of one of the principal stresses at the points of tangency. Stress is a second-rank tensor which components satisfy differential equations of equilibrium, DEE, and certain constitutive equations. The latter constitute a broad class and some examples are found in engineering: 
- in elasticity, the laplacian applied to the first invariant of the stress tensor should vanish;

- in ideal plasticity, the deviator of the stress tensor is a constant;

- in granular medium, certain linear relationships between the mean stresses and the stress deviator should be fulfilled;

- in rock mechanics non-linear relationships are frequently used.

This study employs the stress trajectory concept for the case of ideal plasticity in order to build slip grids in plastic zones forming near an excavation.

\section{Mathematical model of damaged zones near long-wall excavations}

\subsection{Assumptions in modelling}

It is accepted that stress field near an excavation can be described by the plane stress condition. This is typical for tunnels, well-bores and other long-wall excavations provided that their axes are oriented along one of the principal stress. It is assumed that the excavation has been made in weak rocks that can be deformed beyond the yielding limit and therefore surrounding rocks can be damaged. It is also assumed that the rockmass in damage zones is in limiting equilibrium. For simplicity, the damage zones are considered to be ideal plastic. Such an assumption is often accepted for coals, e.g. [3], however this simplification is not crucial and can be easily extended for some other rocks staying in limiting equilibrium to take friction and cohesion into account, see [4].The plastic zone is assumed to be bounded and embedded into infinite elastic zone. The boundary between the zones is not known. The present paper does not deal with the identification of the unknown boundary; if necessary this can be performed by the approach suggested in [5].

\subsection{Lamé - Maxwell equations of equilibrium}

For plane problems there are two independent DEE valid at each point of the domain including the boundary between plastic and elastic zones.

$$
\frac{\partial \sigma_{11}}{\partial x_{1}}+\frac{\partial \sigma_{12}}{\partial x_{2}}=0, \quad \frac{\partial \sigma_{12}}{\partial x_{1}}+\frac{\partial \sigma_{22}}{\partial x_{2}}=0
$$

where $\sigma_{k j}$ are stress components in a Cartesian coordinate system $O x_{1} x_{2}$. The gravitational term has been omitted in (1) for convenience, because it can be accounted for by incorporation into boundary conditions.

DEE (1) can be rewritten in terms of principal stresses and principal directions in the Lame-Maxwell form, [3]:

$$
\frac{\partial \sigma_{1}}{\partial s_{1}}+\frac{\sigma_{1}-\sigma_{2}}{\rho_{2}}=0, \quad \frac{\partial \sigma_{2}}{\partial s_{2}}+\frac{\sigma_{1}-\sigma_{2}}{\rho_{1}}=0
$$

Here $\sigma_{k}$ is a principal stress along the $k$-th stress trajectory which radius of curvature is defined as $\rho_{k}^{-1}=\partial \theta / \partial \hat{s}_{k} ; \theta$ is the principal direction (the angle between $\sigma_{l}$ and the $x_{l}$-axis); $s_{k}$ is the arc length along the $k$-th trajectory. In order 
to distinguish different families of the stress trajectories it is assumed that $\sigma_{2} \leq \sigma_{1}$, these families are further referred to as $s_{1}$ and $s_{2}-$ families.

In plastic zone the following governing equation is valid

$$
\frac{\sigma_{1}-\sigma_{2}}{2}=k
$$

where $k$ denotes the yielding limit of the rocks.

The systems (1), (3) or (2), (3) form closed systems of equations and can be solved independently of kinematics equations. The latter is not included in the analysis because it is not required for the statically determined cases as the one considered here.

\subsection{Classical approach}

Classical texts on ideal plasticity, e.g. [6, 7], suggest to rewrite (1) by substituting (3) and using the following relationships

$$
\sigma_{11}=\sigma-k \sin (2 \vartheta), \quad \sigma_{22}=\sigma+k \sin (2 \vartheta), \quad \sigma_{12}=k \cos (2 \vartheta)
$$

where the angle

$$
\vartheta=\theta-\pi / 4
$$

represents the inclination of the $s_{\alpha}$-family of slip lines; another orthogonal family, the $s_{\beta}$-family, has orientations $\theta+\pi / 4$. Notations are shown in Fig. 1 . This leads to the system of $2 \mathrm{PDE}$ with respect to 2 unknowns $\sigma$ and $\vartheta$ :

$$
\begin{aligned}
& \frac{\partial \sigma}{\partial x_{1}}-2 k\left[\cos (2 \vartheta) \frac{\partial \vartheta}{\partial x_{1}}+\sin (2 \vartheta) \frac{\partial \vartheta}{\partial x_{2}}\right]=0 \\
& \frac{\partial \sigma}{\partial x_{2}}-2 k\left[\sin (2 \vartheta) \frac{\partial \vartheta}{\partial x_{1}}-\cos (2 \vartheta) \frac{\partial \vartheta}{\partial x_{2}}\right]=0
\end{aligned}
$$

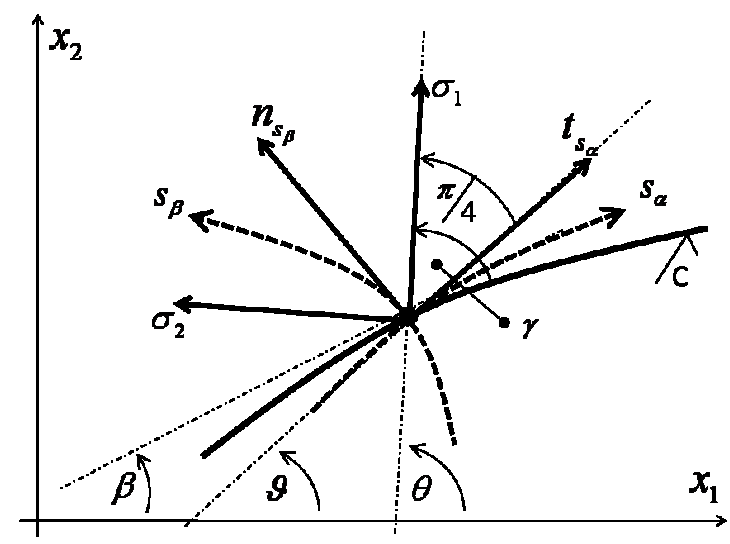

Figure 1: $\quad$ Notations: $\beta$ is the angle of the tangent to the contour $C$ with $x_{I}$ axis, $\gamma$ is the angle between the tangent to the contour and the orientation of the first principal stress $\sigma_{l} . t_{s \alpha}$ and $n_{s \alpha}$ show the tangent and normal to the slip lines $s_{\alpha}$ and $s_{\beta}$. 
After simple transformation of (6) one finds the Henckey integrals and differential equations for determination of the slip grid [5]

$$
\begin{aligned}
& \frac{d x_{2}}{d x_{1}}=\tan \vartheta, \quad \frac{\sigma}{2 k}-\vartheta=\text { const, for } \alpha \text {-family; } \\
& \frac{d x_{2}}{d x_{1}}=-\cot \vartheta, \quad \frac{\sigma}{2 k}+\vartheta=\text { const, for } \beta \text {-family; }
\end{aligned}
$$

After that (7) is solved by using the finite difference method.

The classical Cauchy's problem is solved within a characteristic triangle which boundaries are formed from the contour as the hypotenuse and characteristic lines as its legs. The legs of the triangle can be taken as boundaries for solving the Riemann - Goursat problem providing the solution when boundaries are characteristic lines of different families. By solving this problem the integral surface can be considerably extended.

\subsection{STEM approach}

STEM solves the Lame-Maxwell DEE's in the following form

$$
\frac{\partial \psi}{\partial s_{1}}+\frac{\partial \theta}{\partial s_{2}}=0 \quad ; \quad \frac{\partial \psi}{\partial s_{2}}+\frac{\partial \theta}{\partial s_{1}}=0
$$

where $\psi=\sigma / 2 k$.

Its evident that the $2^{\text {nd }}$ order PDE has the form

$$
\frac{\partial^{2} \theta}{\partial s_{1}^{2}}-\frac{\partial^{2} \theta}{\partial s_{2}^{2}}=0
$$

It should be noted that mean stresses ( $\sigma$ and $\psi$ ) and the angle $\vartheta$ also satisfy this equation. This forms the basis for using alternating Cauchy problems for the slip grids and stress trajectories as illustrated in the next section.

To formulate BVP in terms of principal direction, $\theta$ and derivatives of $\theta$ are needed

$$
\left[\begin{array}{c}
\frac{\partial \psi}{\partial t} \\
\frac{\partial \psi}{\partial n}
\end{array}\right]+\left[\begin{array}{cc}
-\sin 2 \gamma & \cos 2 \gamma \\
\cos 2 \gamma & \sin 2 \gamma
\end{array}\right]\left[\begin{array}{c}
\frac{\partial \theta}{\partial t} \\
\frac{\partial \theta}{\partial n}
\end{array}\right]=\left[\begin{array}{l}
0 \\
0
\end{array}\right]
$$

The decomposition of the derivatives along $s_{1}$ and $s_{2}$-families from (8) into their normal and tangential derivatives has the form

$$
\frac{\partial}{\partial s_{1}}=\cos \gamma \frac{\partial}{\partial t}+\sin \gamma \frac{\partial}{\partial n} \quad, \quad \frac{\partial}{\partial s_{2}}=-\sin \gamma \frac{\partial}{\partial t}+\cos \gamma \frac{\partial}{\partial n}
$$

Using equation (10) the unknown normal derivatives of stresses and orientations can be calculated.

Nodes of the next layers are found using the properties of characteristics, one of which states that characteristics form an orthogonal grid; characteristics of 
different families form an orthogonal system of lines. Intersections of these different families create a set of nodes for the next layer of the grid.

$$
z_{j}^{k+1}=z_{j}^{k}+\delta_{j}^{S_{1}} e^{i \theta_{j}^{k}} \quad, \quad z_{j}^{k+1}=z_{j+1}^{k}+\delta_{j+1}^{s_{2}} e^{i\left(\theta_{j+1}^{k}+\frac{\pi}{2}\right)}
$$

Notations are shown in Fig. 2. In formulas (12) the subscript refers to the node while the superscript specifies the layer number (layer $k$ is known, layer $k+1$ is unknown); $\delta_{j}$ represents the distance between nodes of the layer $k$ and $k+1$ at position $j$ or $j+1$ along the $s_{1}$ or $s_{2}$ characteristic.

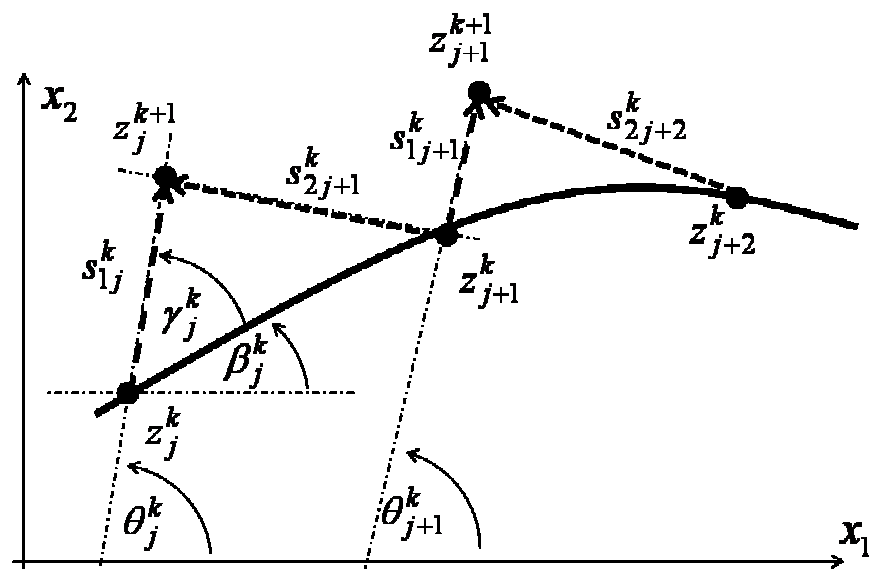

Figure 2: Determination of coordinates and visualization of angles.

At this point the normal derivatives of orientations and stresses as well as the tangential ones are determined and the change of the orientations and stresses along characteristics can be computed using (11). These terms are essential for computing the unknown values in the new layers, for which the Taylor's expansion was used

$$
\theta_{j}^{k+1}=\theta_{j}^{k}+\left.\left|z_{j}^{k+1}-z_{j}^{k}\right| \cdot \frac{\partial \theta}{\partial s_{1}}\right|_{j} ^{k} \quad, \quad \psi_{j}^{k+1}=\psi_{j}^{k}+\left.\left|z_{j}^{k+1}-z_{j}^{k}\right| \cdot \frac{\partial \psi}{\partial s_{1}}\right|_{j} ^{k}
$$

\subsection{Combining STEM and Kachanov}

Classical approach developed for example by Kachanov [7] gives the solution only inside the characteristic triangle stating the solution outside cannot be obtained.

Boundary conditions from the classical approach can be used in STEM to demonstrate the equivalence between these two approaches. Slip Grid (SG) is obtained by using classical solution of Cauchy's problem for the given noncharacteristic boundary while Stress Trajectories (ST) for the corresponding boundary are found by STEM. By rotating the stress orientations in the ST Grid by $-\pi / 4$, orientations of shear stresses are found at every node. 
Solution of the Cauchy problem using STEM gives a characteristic triangle again, which is different from that obtained in the classical approach. The boundaries of this triangle can be used in the next step as new initial boundaries. Bearing in mind that ST Grid was obtained using STEM the following needs to be brought in attention. Boundaries of the triangle are characteristic lines which is why they would form a characteristic boundary for ST. Rotating the orientations on this boundary by $-\pi / 4$ forms a non-characteristic boundary for the slip lines. This new boundary can be then easily used for classical solution.

These two approaches can be alternated to move away from the initial boundary.

\section{Numerical examples}

\subsection{Tunnel in weak rocks}

As an example of the proposed technique, let us consider a tunnel of the geometry shown in Fig. 3.

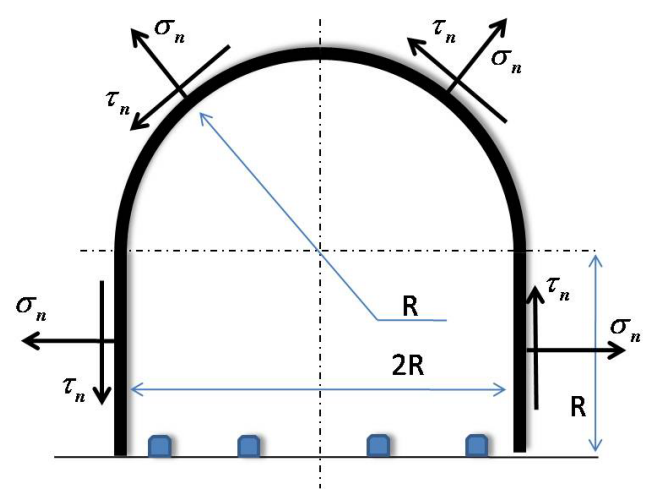

Figure 3: $\quad$ Tunnel in weak rocks ( $\sigma_{n}$ and $\tau_{n}$ are present to model the effects of the supports).

The $\mathrm{BC}$ are chosen as follows

$$
\sigma_{n}=c \quad, \quad \tau_{n}(s)=a s+b
$$

where $a b$ and $c$ are certain piecewise constant coefficients different from linear and circular parts of the boundary.

\subsection{Results}

The following results have been obtained for the classical solution of Cauchy's problem (using Kachanov's approach); solution by STEM; comparison of STEM approach and classical approach. Due to symmetry the problem has been solved for the left half of tunnel's boundary as shown in Fig. 3. 


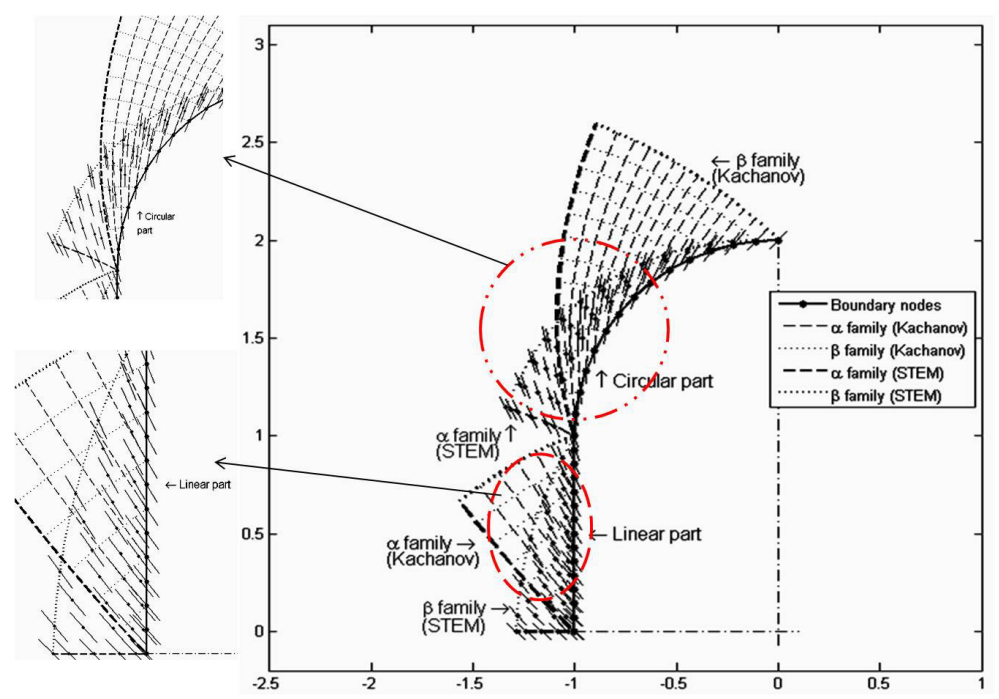

Figure 4: $\quad$ Classical solution (Slip Grid) vs. STEM solution (ST Grid).

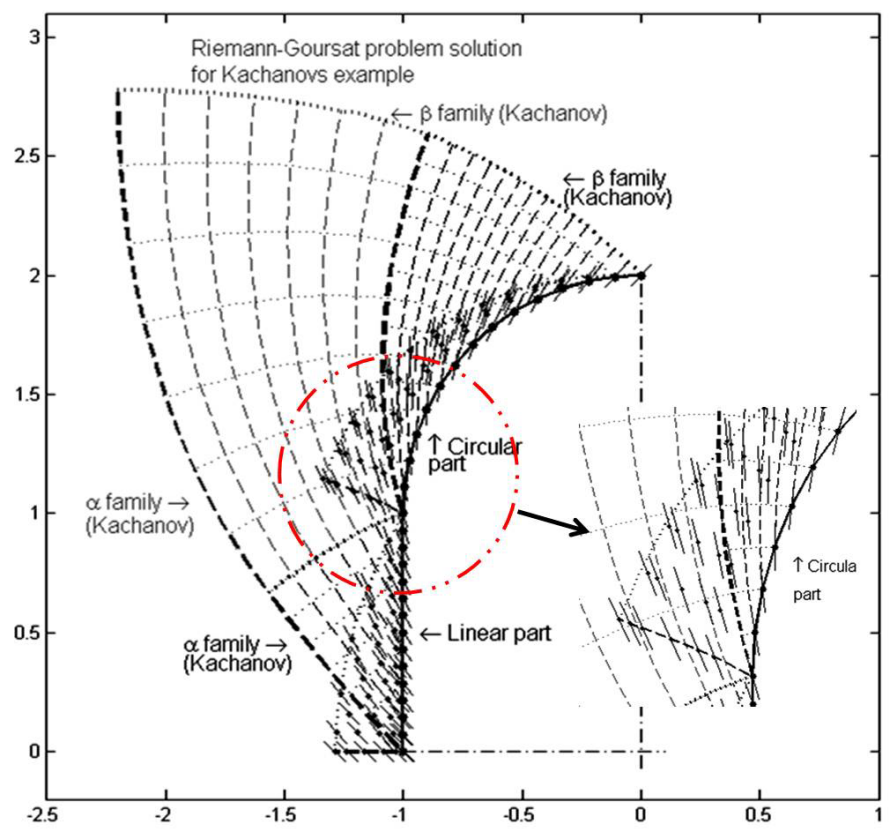

Figure 5: $\quad$ Extended solution using Riemann - Goursat problem (Slip Grid) versus STEM solution (ST Grid). 


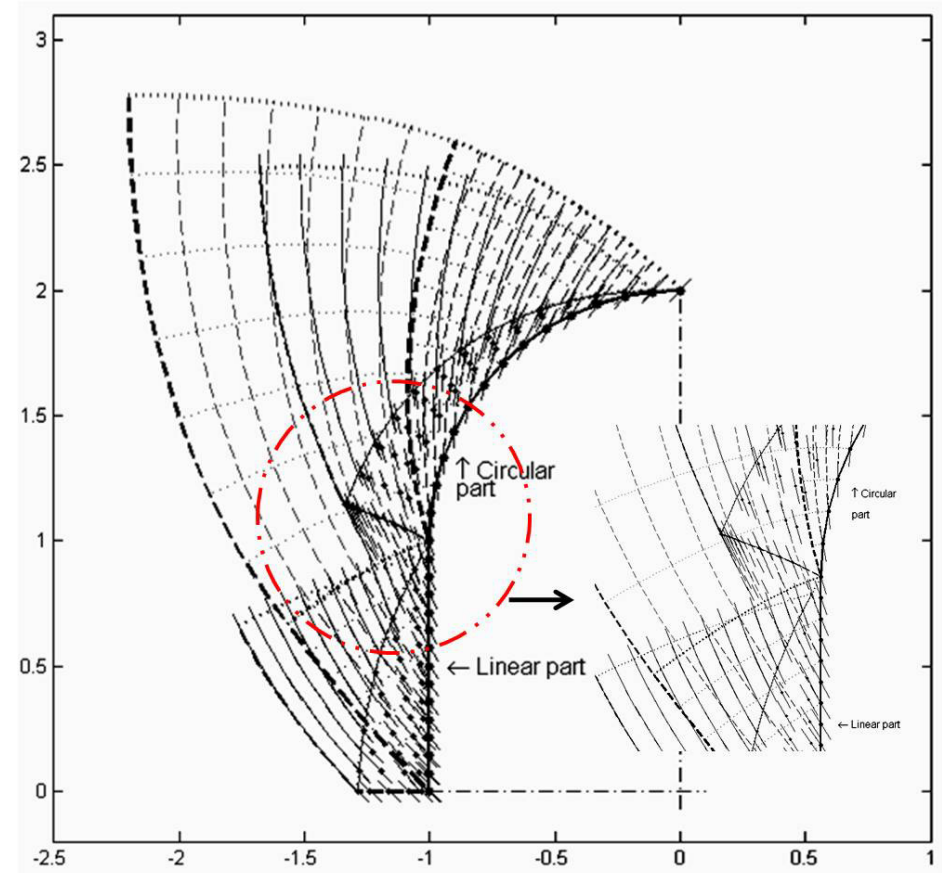

Figure 6: $\quad$ Solution of Cauchy - Riemann problem (Slip Grid) and extended solution by STEM.

\section{Conclusions}

It is shown that slip grid in plastic zones near long-wall excavations can be built by solving the Cauchy boundary value problems alone without using the Goursat problem for the determination of slip lines in intermediate zones. It is shown that the Cauchy problem for the determination of stress trajectory patterns can be formulated instead of the Goursat problem. Once the stress trajectory field is determined the slip grid becomes known by simple rotation of stress orientations. The approach also allows one to extend solutions beyond the characteristic triangle by using the boundary of the characteristic triangle as a new boundary for building stress trajectories and visa versa to build the slip grid from the boundary of ST grid. Good agreement with the classical solutions have been observed for particular cases of geometry and boundary conditions.

\section{Acknowledgement}

The authors acknowledge the support of EPSRC through Research Grant $\mathrm{EP} / \mathrm{E} 032494 / 1$. 


\section{References}

[1] Galybin, A.N. 2007. Introduction of STEM for stress analysis in statically determined bodies. WIT Transactions on Modelling and Simulation, 44, WIT Press, Southampton, UK, 79-88;

[2] Frocht, M.M. Photoelasticity, Vol. 1. Wiley, New York, 1941;

[3] Khistianovich S.A., Salganik, R.L., 1983; Several basic aspects of the forming of sudden outbursts of coal (rock) and gas; 5th Congress International Congress on Rock Mechanics, Melbourne; pp. E41-E50

[4] Goodman, R.E. Introduction to Rock Mechanics. J Wiley, New York, 1980.

[5] Cherepanov, G. P. 1963. On a method of solving the elasto-plastic problem Journal of Applied Mathematics and Mechanics, 27 (3), 644-655

[6] Hill, R.; The Mathematical Theory of Plasticity; Oxford, Clarendon Press; 1950 ;

[7] Kachanov, L.M.; Fundamentals of Theory of Plasticity; Published: Foundations of the theory of plasticity, Amsterdam, 1971; 